\title{
Trions in bulk LiF and at the LiF(001) surface
}

\author{
Deilmann, Thorsten
}

\section{Published in:}

Physical Review B

Link to article, DOI:

10.1103/PhysRevB.98.165105

Publication date:

2018

Document Version

Publisher's PDF, also known as Version of record

Link back to DTU Orbit

Citation (APA):

Deilmann, T. (2018). Trions in bulk LiF and at the LiF(001) surface. Physical Review B, 98(16), [165105]. https://doi.org/10.1103/PhysRevB.98.165105

\section{General rights}

Copyright and moral rights for the publications made accessible in the public portal are retained by the authors and/or other copyright owners and it is a condition of accessing publications that users recognise and abide by the legal requirements associated with these rights.

- Users may download and print one copy of any publication from the public portal for the purpose of private study or research.

- You may not further distribute the material or use it for any profit-making activity or commercial gain

- You may freely distribute the URL identifying the publication in the public portal

If you believe that this document breaches copyright please contact us providing details, and we will remove access to the work immediately and investigate your claim. 


\title{
Trions in bulk $\mathrm{LiF}$ and at the $\operatorname{LiF}(001)$ surface
}

\author{
Thorsten Deilmann* \\ CAMD, Department of Physics, Technical University of Denmark, DK-2800 Kongens Lyngby, Denmark
}

(Received 14 August 2018; published 3 October 2018)

\begin{abstract}
We study the neutral and charged optical excitations (excitons and trion) in the prototypical wide-gap insulator $\mathrm{LiF}$ using the first-principles $G W / \mathrm{BSE}$ approach and its extension for trions. In addition to neutral excitons we find bound trion states with a trion binding energy of 20-30 meV. At the same time the lowest bright exciton and trion are well separated by about $400 \mathrm{meV}$ in the absorption spectrum both for the bulk and the (001) surface crystal. Even though the charged trions are slightly more extended compared to their neutral counterparts, they remain localized on a few atoms only and keep the Frenkel-like character of the neutral excitons. With such strongly localized trions, LiF stands out from currently investigated trionic materials and highlights itself for further studies.
\end{abstract}

DOI: 10.1103/PhysRevB.98.165105

\section{INTRODUCTION}

The optoelectronic properties of matter are of key interest for next-generation technology. Their applications include light generation (e.g., using light-emitting devices such as lasers), information transfer (e.g., with optical fibers), and light collection and transformation to energy (e.g., using photodetectors or solar cells). For such different applications, materials with a variety of different properties are needed. Moderate band gaps are required for visible light, which has inspired intensive research into two-dimensional (2D) materials like transition metal dichalcogenides [1]. Another class with distinctly different materials with remarkable properties are insulating alkali halides, and among them LiF, with a huge bulk band gap of about $14.2 \mathrm{eV}$ [2] and large exciton binding energies (the lowest bright peak shows up at about $12.7 \mathrm{eV}$ [3]), is of particular interest. For applications, the surface of a material can play an important role. Khemliche et al. $[4,5]$ have shown that the $\operatorname{LiF}(001)$ surface can be doped effectively by $\mathrm{Ne}^{+}$bombardment and that signatures of trions can be observed thereafter.

Until now the description of trions in $\mathrm{LiF}$ has been limited to the approximative multiconfiguration self-consistent field calculations of small clusters [6]. Furthermore, the usage of a Wannier-Mott model (i.e., employing $k$-dependent envelope functions) is questionable for $\mathrm{LiF}$ because excitations are strongly localized [7]. We note that this Frenkel-like behavior of the excitons and trions is in contrast to other materials, such as 2D transition metal dichalcogenides [8], 1D nanostructures like carbon nanotubes or carbon nanoribbons $[9,10]$, or quantum dots $[11,12]$. In a number of theoretically studies [7,13-15] the electronic and neutral optical properties of $\mathrm{LiF}$ turned out to be well described by the $G W / \mathrm{BSE}$ approach $[16,17]$. In this study we will describe neutral excitonic and charged trionic effects on equal footing, employing our recently developed $a b$ initio approach $[9,18]$. This method is

\footnotetext{
*thorsten.deilmann@wwu.de
}

applied to the 3D bulk LiF crystal (Sec. II) as well as the $\mathrm{LiF}(001)$ surface (Sec. III).

\section{THE LiF BULK CRYSTAL}

Bulk LiF has a rocksalt crystal structure with an experimental lattice constant of $4.03 \AA$ [19], which we employ in this study. As starting point for the investigation of the optical properties, the electronic band structure (see Fig. 1) is calculated. Compared to the experimental value of $14.2 \mathrm{eV}$, the direct band gap at $\Gamma$ is drastically underestimated in LDA, with $8.63 \mathrm{eV}$ only. For our DFT calculations we use norm-conserving pseudopotentials in the Kleinman-Bylander form [20,21] and expand the wave functions in a basis of localized atom-centered Gaussian orbitals of $s, p, d$, and $s^{*}$ symmetry [22]. When employing the $G W$ approximation [23,24] a drastic increase of the band gap to about $14.28 \mathrm{eV}$ is observed. We note that this result is achieved by employing Gaussian orbitals (GO) to calculate the two-point quantities, which are in good agreement with the result of $14.33 \mathrm{eV}$ employing plane waves (PWs) (extrapolated to an infinite basis size). ${ }^{1}$ In the experiment a gap of about $14.2 \mathrm{eV}$ [2] is observed, which agrees well with our $G W$ result as well as previous calculations [7,13-15]. In Fig. 2 the optical absorption of neutral bulk LiF is shown in black. To evaluate this, we solve the Bethe-Salpeter equation

$$
\left(\epsilon_{\mathbf{c}}-\epsilon_{\mathbf{v}}\right) A_{\mathbf{v} \mathbf{c}}^{S}+\sum_{\mathbf{v}^{\prime} \mathbf{c}^{\prime}}\left\langle\mathbf{v c}\left|K_{\mathrm{eh}}\right| \mathbf{v}^{\prime} \mathbf{c}^{\prime}\right\rangle A_{\mathbf{v}^{\prime} \mathbf{c}^{\prime}}^{S}=\Omega^{S} A_{\mathbf{v c}}^{S},
$$

in which $\epsilon_{\mathbf{v} / \mathbf{c}}(\mathbf{v} / \mathbf{c}$ denotes the band $v / c$ and $\mathbf{k}$ point $)$ are the quasiparticle energies of valence and conduction band electrons, $K_{\mathrm{eh}}$ is the electron-hole kernel (in the $G W$ approximation) including the screened direct and the bare exchange

\footnotetext{
${ }^{1}$ In both calculations we employ a scissors operator before the RPA screening and the $G W$ self-energy operator are evaluated, to anticipate the opening of the gap in a self-consistent approach.
} 


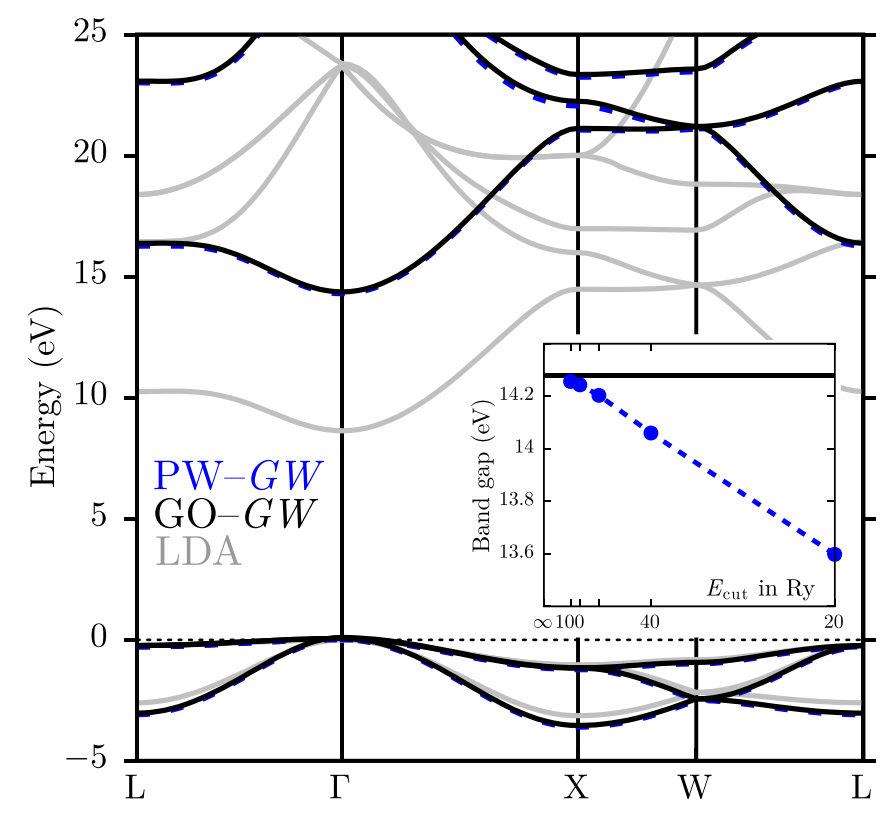

FIG. 1. Band structure of LiF bulk in LDA (grey) and $G W$ with a Gaussian orbital (GO, black) and plane-wave basis set (PW, blue). The inset shows the convergence of the band gap with the cutoff energy $E_{\text {cut }}$ in comparison to the GO calculation. For all calculations a $4 \times 4 \times 4 k$ mesh is used.

interaction, and $\Omega^{S}$ and $A_{\mathbf{v c}}^{S}$ are the exciton energies and coefficients. We find the lowest-energy exciton peak at $12.77 \mathrm{eV}$ with a large exciton binding energy of $1.51 \mathrm{eV}$. Again this is in good agreement with the experimentally observed value of $12.7 \mathrm{eV} \mathrm{[3]} \mathrm{and} \mathrm{previous} \mathrm{theoretical} \mathrm{studies} \mathrm{[16,17].} \mathrm{This}$ bright exciton peak results from a spin singlet exciton. In addition, we note that the lowest dark transition results from a triplet exciton and is found at $12.37 \mathrm{eV}$.

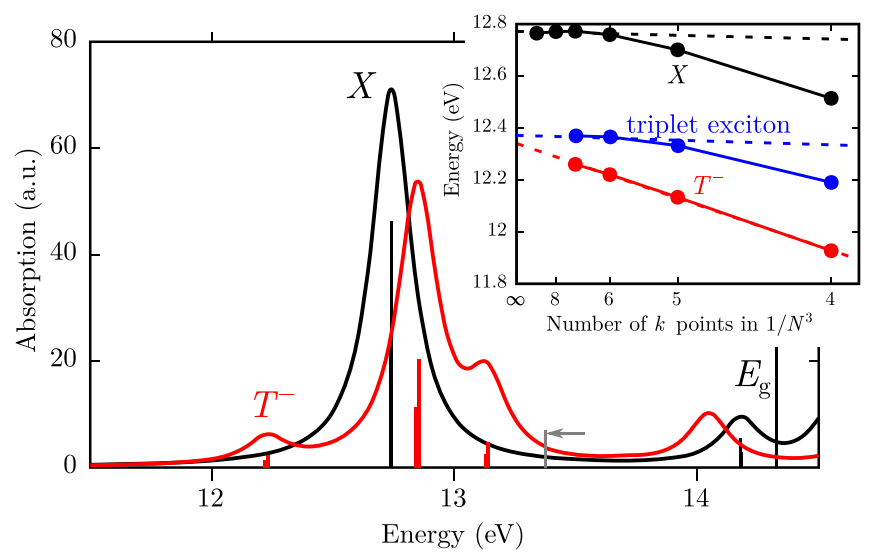

FIG. 2. Optical properties of bulk LiF below the band gap $E_{\mathrm{g}}$. The neutral excitons (black) are compared to negatively charged trions (red). We note that the spectral weights of excitons and trions cannot be compared directly (see main text). Above $13.3 \mathrm{eV}$ (grey arrow) the spectrum is calculated with the Haydock recursion scheme. The inset shows the convergence of the energetically lowest bright exciton, the energetically lowest optically dark triplet exciton (blue), and the trion with respect to the employed $k$ point mesh. All curves are linear extrapolations.

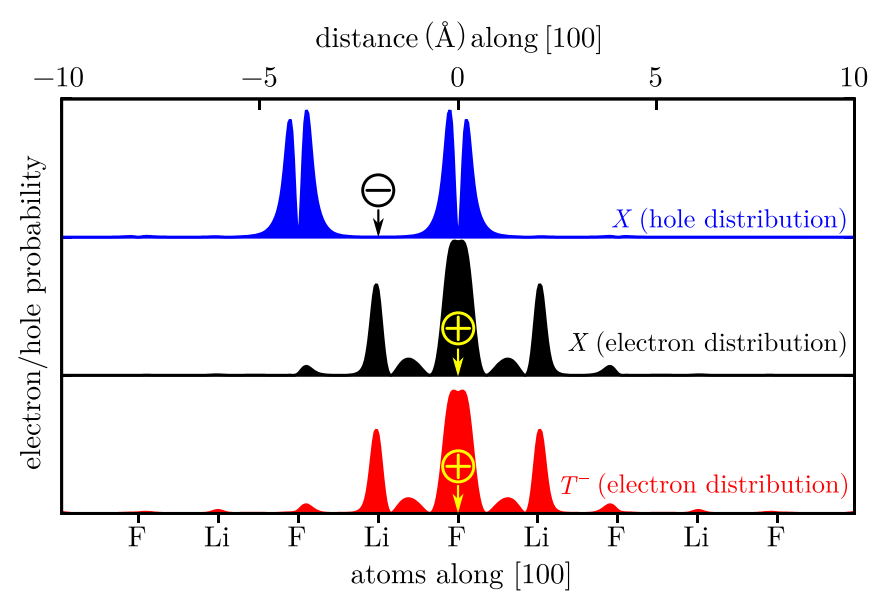

FIG. 3. Electron/hole probability of the exciton $X$ and trion $T^{-}$ along the [100] direction of the bulk crystal. The blue curve shows the hole probability of the exciton for an electron localized at a $\mathrm{Li}$ atom; the black curve shows the electron probability if the hole is localized on a F atom. In red the corresponding electron distribution of the trion is shown after averaging over the second electron.

In red (Fig. 2) we show the result for a negative doping. We note in passing that the spectrum for positive doping is nearly identical and is not further discussed here. To calculate this spectrum we solve

$$
\begin{aligned}
& \left(\epsilon_{\mathbf{c}_{1}}+\epsilon_{\mathbf{c}_{2}}-\epsilon_{\mathbf{v}}\right) A_{\mathbf{v c}_{1} \mathbf{c}_{2}}^{T}+\sum_{\mathbf{v}^{\prime} \mathbf{c}_{1}^{\prime} \mathbf{c}_{2}^{\prime}}\left\langle\mathbf{v} \mathbf{c}_{1} \mathbf{c}_{2}\left|K_{\text {eeh }}\right| \mathbf{v}^{\prime} \mathbf{c}_{1}^{\prime} \mathbf{c}_{2}^{\prime}\right\rangle A_{\mathbf{v}^{\prime} \mathbf{c}_{1}^{\prime} \mathbf{c}_{2}^{\prime}}^{T} \\
& =\Omega^{T} A_{\mathbf{v c}_{1} \mathbf{c}_{2}}^{T},
\end{aligned}
$$

which now incorporates an additional electron $\mathbf{c}_{2}$ and the electron-electron-hole interaction kernel $K_{\text {eeh }}$ (see Ref. [9] for further details). The energetically lowest peak is found at $12.34 \mathrm{eV}$ and thus the corresponding trion obtains a large energetic distance of $430 \mathrm{meV}$ compared to the corresponding bright exciton. ${ }^{2}$ We underline that the trion binding energy with reference to the triplet exciton is much smaller, with a value of $30 \mathrm{meV}$ only. To get deeper into the character of the excitations, we evaluate the spatial extent of the exciton and trion wave functions in real space (Figs. 3 and 4). Both low-energy excitons (singlet and triplet) and the trion $T^{-}$ have a similar character in reciprocal space; i.e., they are located around $\Gamma$ and arise from transitions between the spindegenerated uppermost valence band and lowermost conduction band (Fig. 1). The valence bands show strong F $(2 p)$ character while the conduction band can be characterized as $\mathrm{Li}(2 s)$. Therefore, the energetically lowest excitation exhibit an electron on $\mathrm{Li}$ and a hole on $\mathrm{F}$.

In Fig. 3 we have fixed the electron on the lithium atom (upper panel) or the hole on the central fluorine atom (middle panel) and plotted the hole or electron distribution, respectively. In good agreement with previous studies $[7,15]$ we observe that the corresponding hole or electron mostly resides on the first neighboring atoms. We note that the two-atomic

\footnotetext{
${ }^{2}$ We note that all binding energies are extrapolated to an infinite $k$ mesh; see inset in Fig. 2.
} 


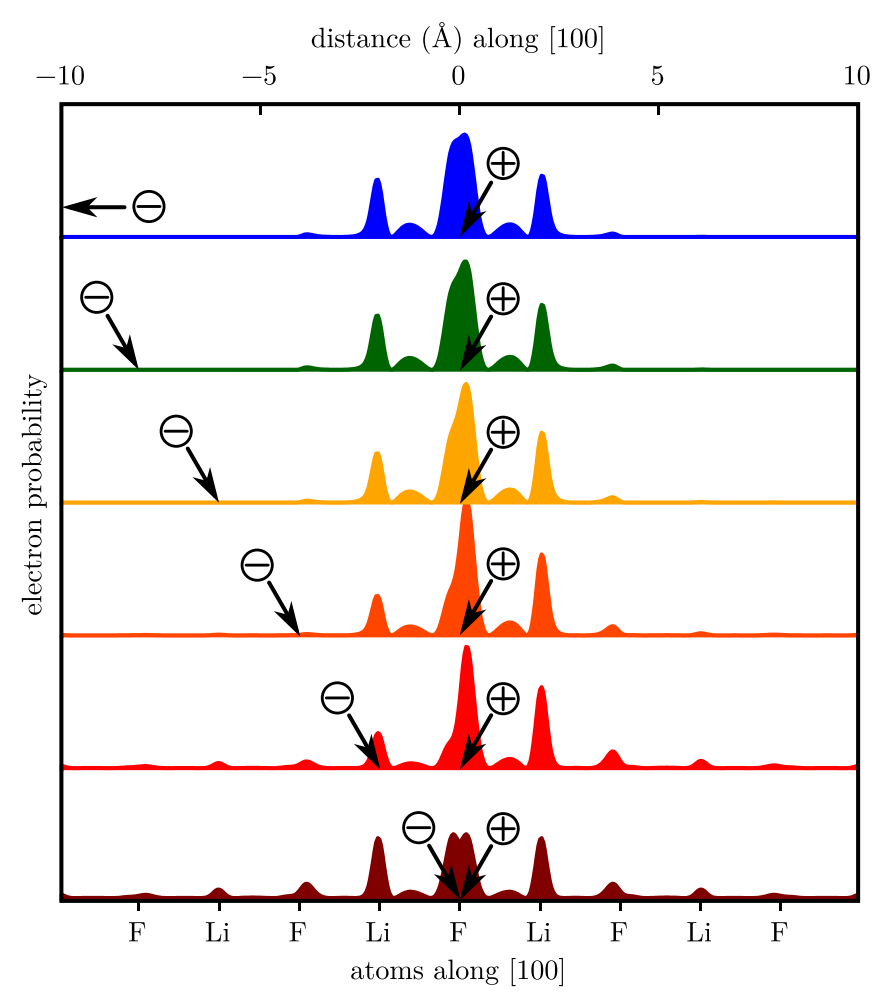

FIG. 4. Electron distribution of the trion for different positions of the second electron compared to the centered hole. The probability is shown on a line along the [100] direction (see Fig. 3).

unit cell is extended by a $10 \times 10 \times 10$ mesh to allow for the description of excitations larger than the unit cell. For the trion $T^{-}$(lower panel) we also fix the hole on a fluorine atom and evaluate the distribution of the first electron while integrating over the second electron (for fixing the second electron, see

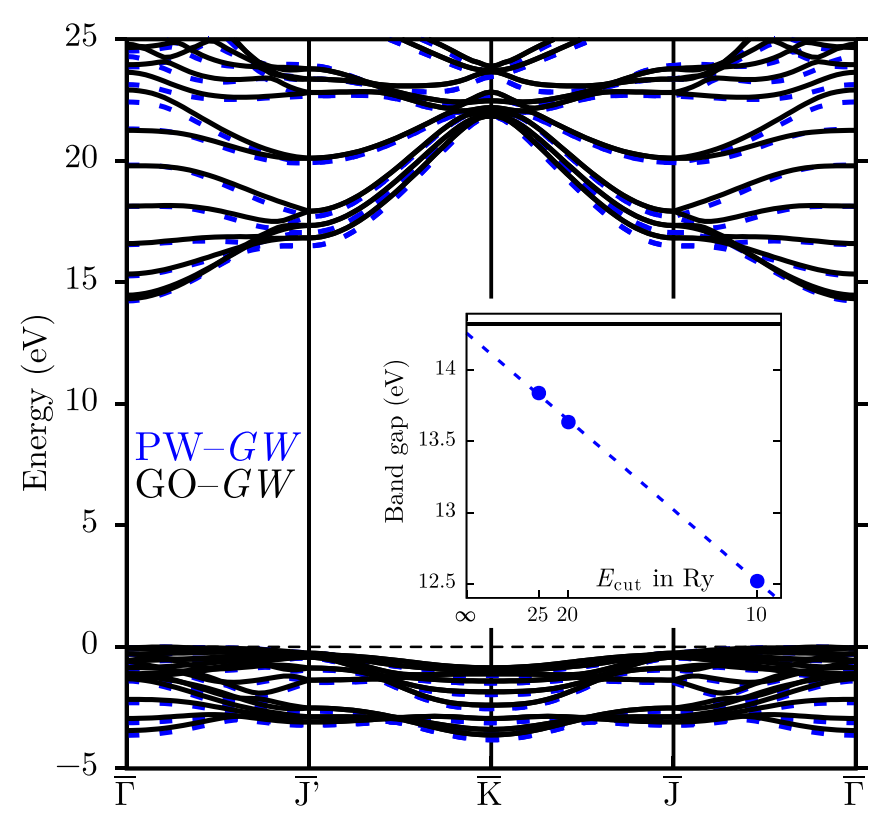

FIG. 5. $\mathrm{LiF}(001)$ band structure using $G W$ with GO (black) and PW (extrapolated, blue). The inset shows the gap at $\Gamma$ with respect to the employed PW cutoff and its linear extrapolation.

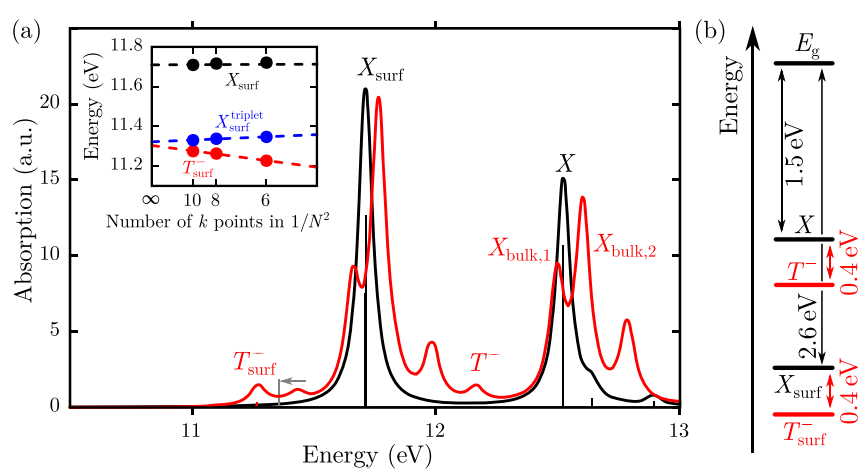

FIG. 6. (a) Optical absorption of the $\operatorname{LiF}(001)$ surface due to neutral excitons (black) and negative trions (red). The spectrum above $11.5 \mathrm{eV}$ (grey arrow) is calculated by the Haydock recursion scheme. The inset shows the convergence with respect to the employed $k$ point mesh. (b) Schematic diagram for the lowest optical bright exciton and trion states for $\mathrm{LiF}$ bulk and surface.

below). In comparison to the exciton, we observe a slightly larger amplitude on the first, second, and third neighbors. The larger extent of the trion arises due to the additional electronelectron repulsion. However, the trion is clearly localized, especially in contrast to the character of trions observed in 2D or 1D materials $[10,18]$.

In the next step we discuss different snapshots which lead to the electron distribution in Fig. 3. In Fig. 4 we keep the hole fixed on the fluorine atom and plot the electron distribution of the first electron when varying the position of the second. If the second electron is far away from the hole, the distribution of the other electron is nearly identical to those observed in an exciton (compare Fig. 3, middle panel) with a minor asymmetry due to the electron-electron repulsion. When the second electron is closer to the hole (lower two panels) the electron distribution is widened. Significant probability is found up to the third neighbor (with respect to the fixed hole). This broadened probability explains the previously discussed distribution when integrating over the second electron (i.e., averaging over the given and many further snapshots).

\section{THE LiF(001) SURFACE}

After investigating the bulk we now turn to the $\mathrm{LiF}(001)$ surface. As previously discussed [7], only minor relaxations are expected at the LiF surface, and in the current study we employ the ideal surface. The surface band structure for a six-layer slab is shown in Fig. 5. We find a surface band gap of $14.32 \mathrm{eV}$ employing GO. ${ }^{3}$ Hence the band gap is nearly identical to the bulk material.

Figure 6(a) shows the optical properties of the $\operatorname{LiF}(001)$ surface calculated with Eqs. (1) and (2). In addition to the previously discussed exciton and trion peaks, further states $X_{\text {surf }}$ and $T_{\text {surf }}^{-}$are observed. The exciton $X_{\text {surf }}$ can be excited

\footnotetext{
${ }^{3}$ We note that the calculations using PW for the two-point quantities (extrapolated to $14.26 \mathrm{eV}$ ) are computationally demanding for a surface system. Thus we have concentrated on the GO basis when evaluating the electron-hole interaction.
} 


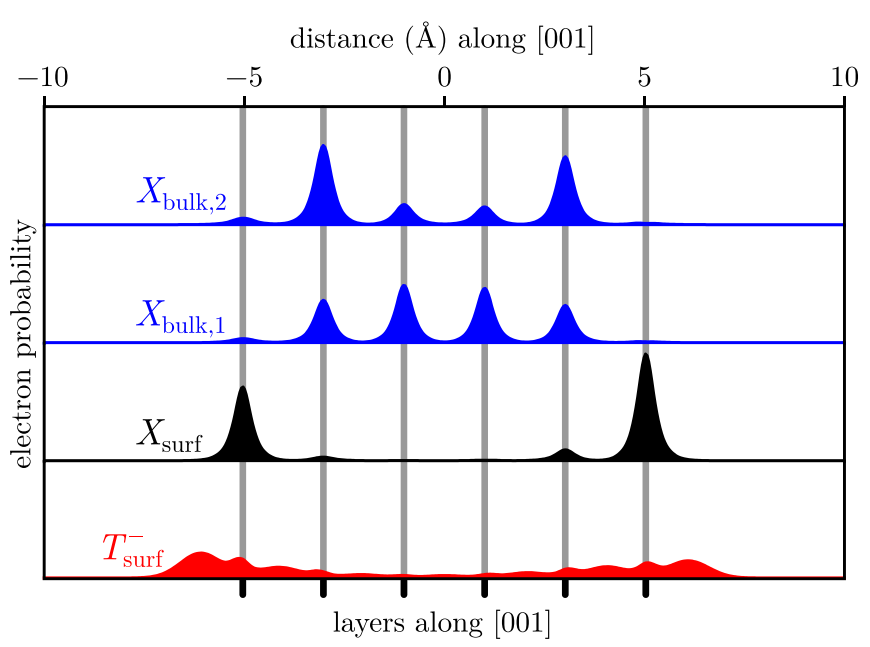

FIG. 7. Integrated electron distribution of the exciton and the trion along a line on the [001] axis. Holes are placed on all $\mathrm{F}$ atoms.

with a photon energy of $11.71 \mathrm{eV}$, while the trion $T_{\text {surf }}^{-}$is found at $11.31 \mathrm{eV}$. The distance of the bright surface singlet exciton and trion is about $400 \mathrm{meV}$, but the surface trion binding energy is only $20 \mathrm{meV}$ when the triplet state is referenced. Thus it is of similar size compared to the values observed in bulk. In addition to these bound states, further resonant states appear in the trion spectrum which are not completely converged. A schematic representation of optical bright peaks in Fig. 6(b) summarizes the prominent bound states below the band gap. The additional exciton/trion binding energy for surface excitation is very similar and results in four bright peaks with distances of about $0.4,0.6$, and $0.4 \mathrm{eV}$. Again we stress that this would suggest large trion binding energies. Incorporating the dark triplet excitons in the discussion, we find relatively small trion binding energies of 20 and $30 \mathrm{meV}$ only, as expected for 3D materials.

Finally we analyze the character of the excited surface states. In reciprocal space both excitons and trions have contributions which reside close to $\bar{\Gamma}$. To investigate the character of the excitations we plot the electron distribution perpendicular to the slab (i.e., along the [001] axis). We fix holes at all fluorine atoms and integrate the electron probability perpendicular to the [001] direction. That means a superposition of the excitations at every fluorine atom is shown.
Due to the surface which breaks the symmetry in the $z$ direction, two excitations close by in energy have been observed at the bulk excitations in Fig. 6. The electron probability is shown in the upper panels of Fig. 7. We observe that both peaks $X_{\text {bulk, } 1}$ and $X_{\text {bulk,2 }}$ are located on the four central layers of the slab. In contrast to this, $X_{\text {surf }}$ and $T_{\text {surf }}^{-}$are both localized at the surface, predominantly at the outermost layer. For the exciton we find a strong $p_{x} / p_{y}$ character, i.e., the excitation occurs in the layer. On the other hand, the surface trion is clearly more delocalized and has an amplitude on states with $p_{z}$ character. Therefore, we can conclude that surface trions are much more sensitive to modifications close to the outermost layer compared to the surface exciton.

Our results of the surface excitons are in good agreement in comparison to previous theoretical studies [15]. The experimental results of Khemliche et al. [4,5] suggest an excitation energy of the trion of about $12.6 \mathrm{eV}$, which is higher compared to the result of $11.3 \mathrm{eV}$. However, the experimental spectrum is very broad and we speculate that it might be a superposition with further states like the bulk trion observed in our study. The theoretical work of Solleder et al. [6] on small LiF clusters has estimated minimal and maximal limits for the trion excitation energy of 10.5 to $12.1 \mathrm{eV}$, which are in reasonable agreement with our findings.

In summary, we have performed first-principles calculations of the quasiparticle band structure and optical spectra of neutral and negatively/positively charged $\mathrm{LiF}$ bulk and surface. With our recently introduced $a b$ initio method we find optically active charged trions both in $3 \mathrm{D}$ bulk LiF and at the $2 \mathrm{D} \mathrm{LiF}(001)$ surface. The large split of $0.4 \mathrm{eV}$ between the lowest energy bright neutral and charged excitation could be mostly traced back to effects of the exchange interaction. The resulting trion binding energy is only $20-30 \mathrm{meV}$. In particular, at the surface the trion is more extended than the exciton and may help to selectively investigate neutral or charged excitations. However, the charged trions remain localized on a few atoms only, in contrast to many other currently studied trionic materials.

\section{ACKNOWLEDGMENT}

T.D. acknowledges financial support from the Villum foundation and helpful comments from Peter Krüger.
[1] K. F. Mak, C. Lee, J. Hone, J. Shan, and T. F. Heinz, Atomically Thin $\mathrm{MoS}_{2}$ : A New Direct-Gap Semiconductor, Phys. Rev. Lett. 105, 136805 (2010).

[2] M. Piacentini, D. W. Lynch, and C. G. Olson, Thermoreflectance of LiF between 12 and $30 \mathrm{eV}$, Phys. Rev. B 13, 5530 (1976).

[3] D. M. Roessler and W. C Walker, Optical constants of magnesium oxide and lithium fluoride in the far ultraviolet, J. Opt. Soc. Am. 57, 835 (1967).

[4] H. Khemliche, J. Villette, A. G. Borisov, A. Momeni, and P. Roncin, Electron Bihole Complex Formation in Neutralization of $\mathrm{Ne}^{+}$on $\mathrm{LiF}(001)$, Phys. Rev. Lett. 86, 5699 (2001).
[5] H. Khemliche, A. G. Borisov, A. Momeni, and P. Roncin, Exciton and trion formation during neutralization of $\mathrm{Ne}^{+}$at a LiF (001) surface, Nucl. Instrum. Methods Phys. Res., Sect. B 191, 221 (2002).

[6] B. Solleder, L. Wirtz, and J. Burgdörfer, Excitation energy and pair correlation function of trions near an LiF surface, Phys. Rev. B 78, 155432 (2008).

[7] M. Rohlfing and S. G. Louie, Electron-Hole Excitations in Semiconductors and Insulators, Phys. Rev. Lett. 81, 2312 (1998).

[8] T. C. Berkelbach, M. S. Hybertsen, and D. R. Reichman, Theory of neutral and charged excitons in monolayer transition metal dichalcogenides, Phys. Rev. B 88, 045318 (2013). 
[9] T. Deilmann, M. Drüppel, and M. Rohlfing, Three-Particle Correlation from a Many-Body Perspective: Trions in a Carbon Nanotube, Phys. Rev. Lett. 116, 196804 (2016).

[10] T. Deilmann and M. Rohlfing, Huge trionic effects in graphene nanoribbons, Nano Lett. 17, 6833 (2017).

[11] I. A. Akimov, A. Hundt, T. Flissikowski, and F. Henneberger, Fine structure of the trion triplet state in a single self-assembled semiconductor quantum dot, Appl. Phys. Lett. 81, 4730 (2002).

[12] M. Bayer, G. Ortner, O. Stern, A. Kuther, A. A. Gorbunov, A. Forchel, P. Hawrylak, S. Fafard, K. Hinzer, T. L. Reinecke, S. N. Walck, J. P. Reithmaier, F. Klopf, and F. Schäfer, Fine structure of neutral and charged excitons in self-assembled In(Ga)As/(Al)GaAs quantum dots, Phys. Rev. B 65, 195315 (2002).

[13] E. L. Shirley, L. J. Terminello, J. E. Klepeis, and F. J. Himpsel, Detailed theoretical photoelectron angular distributions for LiF(100), Phys. Rev. B 53, 10296 (1996).

[14] L. X. Benedict, E. L. Shirley, and R. B. Bohn, Optical Absorption of Insulators and the Electron-Hole Interaction: An ab initio Calculation, Phys. Rev. Lett. 80, 4514 (1998).

[15] N.-P. Wang, M. Rohlfing, P. Krüger, and J. Pollmann, Quasiparticle band structure and optical spectrum of $\operatorname{LiF}(001)$, Phys. Rev. B 67, 115111 (2003).
[16] M. Rohlfing and S. G. Louie, Electron-hole excitations and optical spectra from first principles, Phys. Rev. B 62, 4927 (2000).

[17] G. Onida, L. Reining, and A. Rubio, Electronic excitations: Density-functional versus many-body Green's-function approaches, Rev. Mod. Phys. 74, 601 (2002).

[18] M. Drüppel, T. Deilmann, P. Krüger, and M. Rohlfing, Diversity of trion states and substrate effects in the optical properties of an $\mathrm{MoS}_{2}$ monolayer, Nat. Commun. 8, 2117 (2017).

[19] R. Wyckoff, Crystal Structures, Vol. 1, 2nd ed. (Wiley, New York, 1963).

[20] D. R. Hamann, Generalized norm-conserving pseudopotentials, Phys. Rev. B 40, 2980 (1989).

[21] L. Kleinman and D. M. Bylander, Efficacious Form for Model Pseudopotentials, Phys. Rev. Lett. 48, 1425 (1982).

[22] T. Deilmann, P. Krüger, M. Rohlfing, and D. Wegner, Adsorption and STM imaging of tetracyanoethylene on $\mathrm{Ag}(001)$ : An ab initio study, Phys. Rev. B 89, 045405 (2014).

[23] L. Hedin, New method for calculating the one-particle Green's Function with application to the electron-gas problem, Phys. Rev. 139, A796 (1965).

[24] M. Rohlfing, P. Krüger, and J. Pollmann, Quasiparticle bandstructure calculations for $\mathrm{C}, \mathrm{Si}, \mathrm{Ge}, \mathrm{GaAs}$, and $\mathrm{SiC}$ using Gaussian-orbital basis sets, Phys. Rev. B 48, 17791 (1993). 